\title{
Effets à court terme du décapage de la matière organique sur la croissance et la nutrition d'épinettes noires et de pins gris mis en terre en forêt boréale
}

\author{
par Nelson Thiffault ${ }^{1}$
}

\begin{abstract}
RÉSUMÉ
Le scarifiage contribue à créer des microsites pour le reboisement en mélangeant la matière organique $(\mathrm{MO})$ aux couches superficielles de sol minéral. Toutefois, sur certaines stations boréales caractérisées par des humus épais et un couvert dominé par les plantes éricacées, la compétition semble limiter les avantages du scarifiage. Une solution pourrait être d’enlever complètement la MO par décapage. Notre objectif est de comparer, sur une telle station, les effets à court terme du scarifiage et du décapage sur la croissance de plants de Picea mariana et de Pinus banksiana. Après 3 saisons, nous avons exploré les relations entre la croissance des plants et les groupes d’espèces de végétation concurrente, la profondeur de mise en terre et la distance jusquà lamas de MO le plus proche. Les deux traitements ont eu des effets semblables sur les variables mesurées. La croissance dans les parcelles décapées était indépendante de la distance à la MO. La profondeur de mise en terre a eu un effet nul ou positif sur la croissance des plants. Les plants de Pinus ont poussé beaucoup plus vite que ceux de Picea. Ĺabsence de différence à court terme entre les traitements doit être interprétée avec prudence, compte tenu des effets potentiels à moyen et à long termes de l'exportation de la MO sur les processus nutritionnels et lérosion.
\end{abstract}

Mots-clés : reboisement, plantation, préparation de terrain, Picea mariana, Pinus banksiana

\begin{abstract}
Scarification contributes to creating planting microsites for reforestation by mixing organic matter (OM) with the upper layers of mineral soil. However, on some boreal sites characterized by a thick humus layer and dominated by ericaceous shrubs, competition seems to limit the benefits of scarification. A potential solution would be to remove OM completely through scalping. The objective of our study was to compare the short-term effects of scarification and scalping on the growth of Picea mariana and Pinus banksiana on such sites. After three growing seasons, we explored the relationships between seedling growth, groups of competing vegetation species, planting depth and distance to the nearest OM accumulation. The two treatments had similar effects on these variables. Growth on plots subjected to scalping was independent from the distance to OM. Planting depth had a nil or positive effect on seedling growth. Pinus seedlings largely outgrew Picea seedlings. However, the lack of short-term difference between scarification and scalping must be interpreted with caution, given the potential mid- and long-term effects of OM exportation on soil fertility, erosion and seedling nutrition.
\end{abstract}

Key words : reforestation, plantation, site preparation, Picea mariana, Pinus banksiana

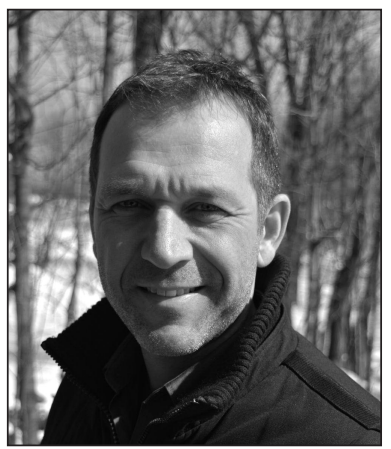

Nelson Thiffault

\section{Introduction}

Laménagement durable des forêts repose notamment sur le maintien de la productivité des écosystèmes, lequel implique que les sites coupés se régénèrent adéquatement après les perturbations (Lieffers et al. 2003). En absence d'une régénération naturelle suffisante, la plantation permet de restaurer la productivité des stations en compensant la distribution ou la densité trop faible des semis naturels (Nyland 2002). Cependant, le succès détablissement des plants de reboisement dépend d'interactions entre le matériel planté (l'essence, le type de plants, le génotype) et les conditions environnementales (Grossnickle 2012). Plus particulièrement, le microenvironnement des plants influence la quantité des ressources disponibles (température de lair et du sol, éléments nutritifs, eau, lumière). Une disponibilité non optimale des ressources crée des stress qui mettent en péril le succès détablissement des plants (Margolis and Brand 1990). Afin d'optimiser l'important investissement associé aux programmes d'amélioration génétique, à la production des plants forestiers et à leur mise en terre (Thiffault et al. 2013), il importe que les microsites de reboisement favorisent des réserves de ressources qui se rapprochent autant que possible des valeurs optimales (Burdett 1990).

Toutefois, les caractéristiques précises des microsites de reboisement qui limitent les stress de plantation sont difficiles à cerner (quantité de débris ligneux, Devine and Harrington

${ }^{1}$ Direction de la recherche forestière, Ministère des Forêts, de la Faune et des Parcs du Québec, 2700, rue Einstein, Québec, QC, G1P 3W8, et Centre d'étude de la forêt, Université Laval, 2405, rue de la Terrasse, Québec, QC, G1V 0A6. Courriel : nelson.thiffault@mffp.gouv.qc.ca 
2007; mélange organique/minéral, Salonius 1983; profondeur de mise en terre, Sutton 1995). De nombreuses études ont documenté les effets du scarifiage sur létablissement des plantations en forêt boréale (p. ex. Prévost et Dumais 2003, Hébert et al. 2014, Henneb et al. 2015). D’autres se sont attardées à la gestion des débris de coupe et à leurs effets sur le microenvironnement des plants (p. ex. Trottier-Picard et al. 2014), ou encore, à leffet de la profondeur de mise en terre sur la croissance (Paquette et al. 2011) et le déchaussement (Sahlén and Goulet 2002). Cependant, beaucoup de stations mésiques du nord-est du Québec (Canada) sont caractérisées par des humus épais qui saccumulent en raison de cycles de feux très longs (Bouchard et al. 2008, Ward et al. 2014). De plus, les peuplements matures sont caractérisés par un sous-bois dominé par les plantes éricacées (notamment le Rhododendron groenlandicum (Oeder) Kron et Judd et le Kalmia angustifolia L.; Thiffault et al. 2015), dont l'abondance semble augmenter à la suite des coupes forestières (Thiffault et Grondin 2003). Dans ces conditions particulièrement contraignantes, le scarifiage par sillons semble moins efficace pour créer des microsites adéquats. En effet, la litière des plantes éricacées est reconnue pour être riche en composés phénoliques qui réduisent directement et indirectement la croissance des conifères (Bradley et al. 1997, Inderjit and Mallik 1999, LeBel et al. 2008). Ainsi, bien que le scarifiage par sillons favorise des températures du sol plus chaudes et stimule la décomposition de la matière organique (Prévost 1992), les propriétés de l'humus forestier associé aux éricacées pourraient limiter les bienfaits escomptés de son mélange avec le sol minéral par le scarifiage. Également, considérant que même un recouvrement relativement faible en Kalmia peut entraîner une diminution significative de la croissance initiale des conifères (Thiffault $e t$ al. 2005), il importe de créer des microsites de plantation dont la surface exempte de l'influence de cette espèce est la plus grande possible (Thiffault et al. 2012). Or, l'efficacité du scarifiage par sillons à créer de bons microsites peut être limitée lorsque les humus forestiers sont très épais (Henneb et al. 2015). Des méthodes plus radicales, tel le hersage forestier, pourraient être envisagées, mais la disponibilité régionale des équipements spécialisés, la présence de sols minces ou la forte pierrosité des stations peuvent en restreindre l'utilisation (Gravel et al. 2015).

Le décapage de la matière organique est une pratique sylvicole généralement proscrite (Prévost et Thiffault 2013) puisque l'enlèvement complet de l'humus forestier prive les microsites d'un capital important déléments nutritifs (Munson et al. 1993, Prescott et al. 2000). Le sol minéral mis à nu est aussi sujet à des variations plus fortes de température que celui recouvert d'humus, en raison des propriétés isolantes de ce dernier (Prévost 1992). De telles variations peuvent favoriser le déchaussement des plants (Goulet 2000), notamment sur les sols avec une forte proportion d'argile. De plus, le réchauffement du sol exposé par décapage peut en réduire le contenu en eau et entraîner des stress hydriques importants pour les plants. Cette situation a été observée dans une station récemment coupée dans le nord-ouest du Québec, caractérisée par un humus de $8 \mathrm{~cm}$ dépaisseur et un couvert de Kalmia, de Vaccinium sp. et de Cladonia sp. (Thiffault et al. 2004). Pourtant, certains sylviculteurs croient que l'enlèvement complet de l'humus pourrait parfois être bénéfique, par exemple dans les régions caractérisées par d’abondantes précipitations annuelles et où les risques de stress hydriques sont moindres.
Les bienfaits de la création d'un environnement exempt de l'influence des éricacées ou de l'humus qui lui est associé pourraient compenser les effets négatifs du traitement. Peu d'études se sont attardées aux effets du décapage complet de la matière organique dans de telles conditions, lesquelles diffèrent du déblaiement d'hiver des débris ligneux sur sol gelé qui maintient une certaine épaisseur de matière organique audessus du sol minéral. La majorité des travaux sur ce thème ont en effet été réalisés dans des écosystèmes de la forêt mixte ou feuillue (p. ex. Frey et al. 2003, Newmaster et al. 2007, Gradowski et al. 2008, Lazaruk et al. 2008, Dumas 2012) et pour la plupart, avec des traitements limités à quelques mètres carrés. Or, en éliminant la couche de matière organique, le scalpage pourrait, dans une certaine mesure, émuler l'effet des feux d'intensité élevée en forêt boréale et donc faire partie d'une stratégie d’aménagement écosystémique (Gauthier et al. 2008). Cependant, les feux de forêts néliminent généralement pas totalement la matière organique; Greene et al. (2007) rapportent une réduction moyenne de $60 \%$ suite à létude de 14 feux à travers le Canada.

Notre objectif dans cette étude est de déterminer l'effet à court terme du décapage de la matière organique jusquau premier horizon minéral sur la nutrition et la croissance de conifères mis en terre, sur une station forestière de la CôteNord dominée par le Kalmia et caractérisée par une épaisse couche de matière organique. Nous vérifions l'hypothèse que la matière organique incorporée au sol minéral par un scarifiage à disques standard constitue une source à court terme d’éléments nutritifs pour les plants mis en terre, et quelle confère aux microsites des caractéristiques favorables à la croissance initiale des plants, incluant la diminution du recouvrement en éricacées. Sur la base de cette hypothèse, nous avons prédit que l'enlèvement complet de l'humus forestier à laide d'un décapage mécanisé créait des microsites plus pauvres en éléments nutritifs que ceux issus d'un scarifiage à disques. Cela se refléterait par des concentrations déléments nutritifs foliaires plus faibles et une croissance moindre des plants mis en terre dans les parcelles décapées. Dans ces mêmes parcelles, nous avons aussi prédit que la croissance des plants diminuerait en fonction de leur distance de l'andain le plus proche (la principale source déléments nutritifs; $\mathrm{McCa}$ vour et al. 2014), et que la profondeur de mise en terre jouait un rôle significatif et positif sur la croissance. Afin de vérifier ces prédictions, nous avons conçu une expérience à léchelle opérationnelle comprenant 2 traitements de préparation de terrain avant la mise en terre de plants dépinette noire (Picea mariana [Mill.] BSP) et de pin gris (Pinus banksiana L.), des essences reconnues pour leur efficacité différente à absorber et utiliser les éléments nutritifs (Reich et al. 1998a).

\section{Matériel et Méthodes}

\section{Station d'étude et dispositif expérimental}

Nous avons établi un dispositif expérimental $\left(50^{\circ} 10^{\prime} 7^{\prime \prime} \mathrm{N}\right.$ $68^{\circ} 44^{\prime} 57^{\prime \prime} \mathrm{O}$ ) dans le domaine bioclimatique de la pessière à mousses du Québec (Saucier et al. 2009), à environ $120 \mathrm{~km}$ au nord-ouest de la ville de Baie-Comeau (Québec, Canada). La station est située sur un dépôt de till de plus d'un mètre d'épaisseur à partir duquel sest développé un sol de type podzolique (Soil Classification Working Group 1998). Le sol, à texture de loam sableux (74 \% sable, $18 \%$ limon, $8 \%$ argile), présente un régime hydrique mésique à subhydrique. Le peuplement original était dominé par lépinette noire avec un 
sous-bois dominé par le Kalmia et a été récolté par coupe avec protection de la régénération et des sols en 2010.

En septembre 2011, nous avons délimité 4 blocs expérimentaux de $60 \times 50 \mathrm{~m}$ chacun, lesquels ont été divisés en 2 parcelles principales de $25 \times 50 \mathrm{~m}$ séparées d'une bande de $10 \mathrm{~m}$. Dans chaque bloc, 2 traitements de préparation de terrain ont été attribués au hasard aux parcelles : (i) scarifiage par sillons; ou, (ii) décapage complet de l'humus forestier jusquau premier horizon minéral. Le scarifiage a été réalisé à l'aide d'un scarificateur de type T26 (Bracke Forest AB, Bräcke, Suède). Il a produit des sillons rectilignes distants de $2 \mathrm{~m}$ chacun, d'une largeur moyenne de $67 \pm 18 \mathrm{~cm}$ (moyenne \pm écart type) et d'une profondeur moyenne de $39 \pm 12 \mathrm{~cm}$, et bordés d'un amas de matière organique mélangée à du sol minéral. Le décapage a été réalisé à l'aide d'un bouteur D7H (Caterpillar, Peoria, Illinois). Les horizons organiques ont été amoncelés en andains de 1 à $2 \mathrm{~m}$ de hauteur au bout des parcelles. Le dispositif expérimental ne comporte aucune parcelle témoin (non soumise à une préparation de terrain), puisque de nombreuses études ont déjà démontré l'importance de la préparation de terrain pour assurer la survie et la croissance initiale des plants mis en terre sur des stations boréales dominées par les éricacées ( $p$. ex. Prévost et Dumais 2003, Thiffault and Jobidon 2006).

En juin 2012, nous avons divisé chaque parcelle principale en 2 sous-parcelles de $25 \times 25 \mathrm{~m}$, auxquelles nous avons attribué au hasard l'essence à planter : épinette noire ou pin gris. Tous les semis avaient été produits dans des récipients de 45 cavités de $110 \mathrm{~cm}^{3}$ chacune, à partir de sources locales de semences. Un échantillon de 50 plants de chaque essence, sélectionnés de manière aléatoire, a été mis de côté avant la mise en terre pour permettre de caractériser les lots ayant servi au reboisement. La hauteur et les biomasses des pins gris étaient supérieures à celles des épinettes noires (sauf pour les rameaux), mais les concentrations foliaires en éléments nutritifs étaient plus élevées chez les épinettes noires (Tableau 1).

Dans chaque sous-parcelle, la mise en terre sest faite selon une grille d'espacement d'environ 2,5 $\times 2,0 \mathrm{~m}$. Dans les parcelles principales scarifiées, les plants ont été mis en terre entre lépaulement et le creux des sillons. Au centre de chaque sous-parcelle, nous avons ensuite établi une parcelle déchantillonnage circulaire de $8 \mathrm{~m}$ de rayon pour englober en moyenne 35 plants à mesurer par unité expérimentale (pour un total de 563 plants pour l'ensemble de l'expérience).

\section{Mesure des plants et de la végétation}

Après la mise en terre, les plants situés dans les parcelles circulaires déchantillonnage ont été identifiés à l'aide d’une fiche métallique et d'un numéro séquentiel, puis mesurés pour leur hauteur initiale $(\mathrm{cm})$, leur diamètre au niveau du sol $(\mathrm{mm})$, de même que pour leur distance jusquà la plante éricacée la plus proche (jusquà un maximum de $150 \mathrm{~cm}$ ). Nous avons aussi mesuré la profondeur de mise en terre, définie comme la distance verticale entre le dessus de la carotte de tourbe et la surface du sol. Une valeur négative signifie que la carotte a été mise en terre sous la surface, et une valeur positive signifie que la carotte nétait pas complètement enfouie. Pour chacun des plants numérotés mis en terre dans les parcelles décapées, nous avons mesuré la distance entre la base du plant et l'amas de matière organique (dans la grande majorité des cas, l’andain le plus proche).

En juillet 2014, soit pendant la $3^{\mathrm{e}}$ saison de croissance des plants depuis leur mise en terre, nous avons inventorié la végétation concurrente croissant dans un rayon de $50 \mathrm{~cm}$ autour de chacun des plants. Ainsi, nous avons visuellement évalué le pourcentage de recouvrement des plantes (par classes de $5 \%$ ) en utilisant les groupes fonctionnels suivants : arbres, graminées, mousses, éricacées, plantes latifoliées, arbustes ligneux, sphaignes, fougères et aulnes. La distance entre les arbres plantés et l'individu de la famille des éricacées le plus proche a aussi été notée (jusquà un maximum de $150 \mathrm{~cm}$ ). En octobre 2014, soit à la fin de la $3^{\mathrm{e}}$ saison de végétation, tous les arbres plantés ont été remesurés pour leur hauteur et leur diamètre au niveau du sol.

\section{Analyses chimiques}

Dans chaque unité expérimentale, nous avons choisi 3 plants au hasard en octobre 2014 et récolté un échantillon foliaire de lannée courante dans le tiers supérieur de leur houppier, de même qu'un échantillon de sol dans un rayon de $10 \mathrm{~cm}$ au pied de leur tige. Les échantillons de sols ont été récoltés de manière à représenter l'environnement d'enracinement des plants, i.e., à une profondeur qui correspondait à la position de la carotte. Les échantillons ont été conservés au frais jusqu’à leur manipulation en laboratoire. Les échantillons foliaires ont été séchés à $65^{\circ} \mathrm{C}$ pendant $48 \mathrm{~h}$, puis broyés pendant 1 min (Pulverisette 0, Fritsch, Idar-Oberstein, Allemagne). Les échantillons de sol ont été séchés à température ambiante, puis broyés à $2 \mathrm{~mm}$. Les concentrations en $\mathrm{C}$ et en $\mathrm{N}$ ont été déterminées par combustion à $1350^{\circ} \mathrm{C}$ à laide d'un appareil TruMac CN Elemental Analyzer (LECO Corporation, St-Joseph, MI, USA) sur des échantillons de $300 \mathrm{mg}$ pour les tissus et de $500 \mathrm{mg}$ pour les sols. Les concentrations en $\mathrm{P}$ et en $\mathrm{K}$ des tissus foliaires ont été déterminées par spec- 
trométrie démission atomique au plasma (ICAP $61^{\mathrm{E}}$, ThermoInstruments, Franklin, MA) suivant une digestion au $\mathrm{H}_{2} \mathrm{SO}_{4}+\mathrm{H}_{2} \mathrm{O}_{2}$ déchantillons de $100 \mathrm{mg}$ (Walinga et al. 1995). Les échantillons de sols $(3 \mathrm{~g})$ ont été soumis à une extraction Mehlich III avant dêtre analysés par spectrométrie démission atomique au plasma (Carter 1993).

\section{Analyses statistiques}

À l'aide des valeurs de dimensions des plants en juin 2012 et en octobre 2014, nous avons calculé leur accroissement absolu (dimensions en octobre 2014 - dimensions en juin 2012) ainsi que leur taux relatif de croissance sur 3 saisons de végétation :

$$
T R C=\frac{\ln X_{A 2014}-\ln X_{P 2012}}{3}
$$

où $\quad T R C=$ taux relatif de croissance; $X=$ hauteur ou diamètre au niveau du sol; A2014 : mesure prise en octobre (automne) 2014; P2012 : mesure prise en juin (printemps) 2012.

Toutes les analyses ont été réalisées à l'aide du logiciel $\mathrm{R}$ v3.0.2 (R Core Team 2013). Les données de hauteur (automne 2014), de diamètre (automne 2014), d’accroissement absolu et de taux relatif de croissance, de même que les données de concentrations foliaires et du sol en éléments nutritifs (automne 2014) ont été soumises à des analyses de variance (ANOVA) pour modèles mixtes à l'aide de la fonction lme de la librairie lme4 (Bates et al. 2015). Les blocs et les interactions impliquant les blocs ont été considérés comme des effets aléatoires; le traitement de préparation de terrain, l'essence et l'interaction préparation de terrain $\times$ essence ont été inclus comme effets fixes. Les pourcentages de recouvrement des divers groupes d’espèces ont été transformés par le sinus inverse de la racine carrée avant dêtre soumis à une ANOVA pour modèles mixtes, avec la préparation de terrain comme seul effet fixe. Les données de distance entre les arbres plantés et les plants d'éricacées les plus proches ont été analysées à l'aide d’un modèle linéaire simple pour données tronquées (modèle Tobit) en utilisant la fonction $\mathrm{vglm}$ de la librairie VGAM (Yee 2008). Nous avons utilisé la fonction $l m$ de la librairie car (Fox and Weisberg 2011) et réalisé des régressions linéaires entre la profondeur de mise en terre et les taux relatifs de croissance en hauteur et en diamètre. Pour les plants mis en terre dans les parcelles décapées, nous avons aussi produit des régressions entre le taux relatif de croissance et la distance entre les plants et l'andain le plus proche.

Afin d'explorer les corrélations entre les variables décrivant les caractéristiques du microsite et les réponses nutritionnelles et de croissance des plants, nous avons soumis les don- nées de nutrition foliaire, de pourcentages de recouvrement de la végétation, de croissance relative en hauteur et de caractéristiques du sol à des analyses en composantes principales à laide de la fonction PCA de la librairie FactoMineR en utilisant les matrices de corrélations (Lê et al. 2008). Les données des deux essences ont été analysées séparément. Pour chaque analyse, nous avons limité notre interprétation aux axes qui présentaient des valeurs propres (eigenvalues) $>1$. Par ailleurs, nous navons inclus que les données de pourcentages de recouvrement des groupes de végétation qui présentaient des valeurs $>0 \%$ dans au moins un des traitements.

\section{Résultats}

Hauteur, diamètre et accroissements

Le taux de survie des plants mis en terre a été très élevé. Seulement 2 arbres parmi ceux plantés étaient morts au terme du suivi de 3 ans : un pin gris dans une parcelle soumise au décapage et une épinette noire dans une parcelle scarifiée. Nous n’avons décelé aucun effet significatif de la préparation de terrain sur la hauteur, le diamètre et l'accroissement absolu et le taux relatif de croissance des plants des 2 essences $(p \geq 0,128$; Tableau 2). Les différences entre les essences ont toutefois été significatives pour l'ensemble de ces variables (tous les $p<0,001$ ), et ce, sans égard au traitement (Préparation de terrain $\times$ Essence, $p \geq 0,068$; Tableau 2 ). Après 3 saisons de croissance, le pin gris présentait une hauteur moyenne de $58,4 \pm 1,5 \mathrm{~cm}$ (moyenne \pm erreur type) et un diamètre moyen de $15 \pm 0,5 \mathrm{~mm}$. Pour sa part, l'épinette noire avait atteint une hauteur moyenne de 40,2 $\pm 1,5 \mathrm{~cm}$ et un diamètre moyen de $8,7 \pm 0,5 \mathrm{~mm}$. Ces dimensions résultaient d'un accroissement absolu en hauteur, en 3 ans, de $48,5 \pm 1,5 \mathrm{~cm}$ pour le pin gris et de $25,7 \pm 1,5 \mathrm{~cm}$ pour l'épinette noire. Pour la même période, l'accroissement absolu en diamètre a été de $11,6 \pm 0,4 \mathrm{~mm}$ pour le pin gris et de $5,7 \pm 0,4 \mathrm{~mm}$ pour l'épinette noire. Les taux relatifs de croissance ont reflété la même tendance (données non présentées).

\section{Végétation concurrente}

De tous les groupes d'espèces, seules les graminées, les mousses, les éricacées et les plantes latifoliées étaient présentes dans l'environnement immédiat des plants. Les pourcentages de recouvrement en graminées et en mousses (valeurs moyennes de $2 \%$ et $15 \%$, respectivement) étaient

Tableau 2. Résultats des analyses de variance (effets fixes) sur les dimensions et la croissance de plants d'épinette noire et de pin gris soumis à 2 traitements de préparation de terrain (scarifiage par sillons ou décapage de la matière organique).

\begin{tabular}{|c|c|c|c|c|c|c|}
\hline \multirow[b]{2}{*}{ Variable } & \multicolumn{2}{|c|}{$\begin{array}{c}\text { Préparation } \\
\text { de terrain }\end{array}$} & \multicolumn{2}{|c|}{ Essence } & \multicolumn{2}{|c|}{$\begin{array}{l}\text { Préparation de } \\
\text { terrain } \times \text { Essence }\end{array}$} \\
\hline & $\mathbf{F}$ & $p$ & $\mathbf{F}$ & $p$ & $\mathbf{F}$ & $p$ \\
\hline Hauteur après 3 saisons de croissance & 1,70 & 0,284 & 123,98 & $<0,001$ & 0,55 & 0,486 \\
\hline Accroissement absolu en hauteur & 1,14 & 0,364 & 209,10 & $<0,001$ & 0,65 & 0,452 \\
\hline Taux relatif de croissance en hauteur & 0,13 & 0,742 & 576,03 & $<0,001$ & 4,95 & 0,068 \\
\hline Diamètre après 3 saisons de croissance & 4,37 & 0,128 & 111,05 & $<0,001$ & 2,85 & 0,142 \\
\hline Accroissement absolu en diamètre & 4,15 & 0,134 & 117,09 & $<0,001$ & 3,45 & 0,113 \\
\hline Taux relatif de croissance en diamètre & 2,09 & 0,244 & 158,47 & $<0,001$ & 3,00 & 0,134 \\
\hline
\end{tabular}




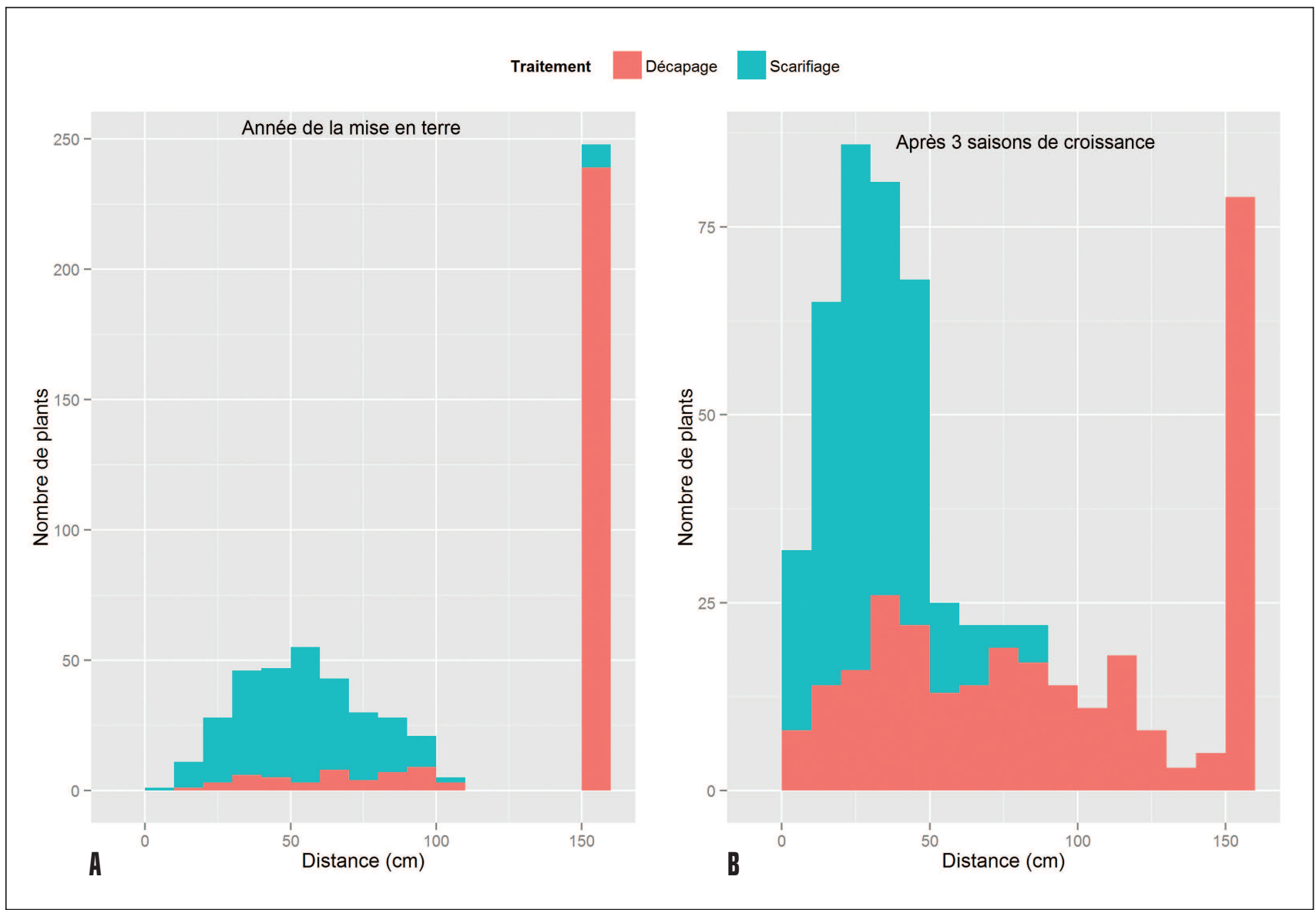

Fig. 1. Distribution du nombre de plants de conifères en fonction de leur distance par rapport au plant d'éricacée le plus proche au moment de la mise en terre (panneau A) et après 3 saisons de croissance (panneau B), jusqu'à une distance maximale de $150 \mathrm{~cm}$.

équivalents dans les parcelles décapées ou scarifiées $\left(\mathrm{F}_{1,3} \leq 0,98, p \geq 0,395\right)$. Le décapage a significativement réduit le pourcentage de recouvrement en plantes éricacées par rapport au scarifiage $\left(\mathrm{F}_{1,3}=118,8, p=0,002\right)$, le faisant passer de $12 \%$ à $1 \%$. Le décapage a aussi réduit le pourcentage de recouvrement en plantes latifoliées par rapport au scarifiage $\left(\mathrm{F}_{1,3}=13,5, p=0,035\right)$, avec des valeurs respectives de $0 \%$ et $3 \%$ pour les 2 traitements. Dans la majorité des microsites des parcelles décapées, le plant déricacée le plus proche au moment de la mise en terre se situait à plus de $150 \mathrm{~cm}$ (Fig. 1A). En comparaison, la distribution dans les parcelles scarifiées était unimodale avec une moyenne de $56 \mathrm{~cm}$ (Fig. 1A). Le modèle Tobit a révélé que la valeur prédite de distance au moment de la mise en terre était de $120 \mathrm{~cm}$ inférieure dans les parcelles scarifiées que dans les parcelles décapées $(Z=-24,6, p<0,001)$. Globalement, l'effet de la préparation de terrain sur la distance était très significatif $(p<0,001)$. Après 3 saisons de croissance dans les parcelles décapées, nous avons observé que la fréquence de la valeur de tronquage « $150 \mathrm{~cm}$ » était toujours beaucoup plus élevée que ce qui pouvait être attendu en fonction du reste de la distribution (Fig. 1B). Pour leur part, les valeurs de distance observées dans les parcelles scarifiées présentaient une moyenne de 31 $\mathrm{cm}$ et aucune occurrence à la valeur de tronquage (Fig. 1B). La valeur prédite de distance après 3 saisons de croissance était de $64 \mathrm{~cm}$ inférieure dans les parcelles scarifiées que dans les parcelles décapées $(Z=-17,5, p<0,001)$. Après 3 saisons de croissance, l'effet de la préparation de terrain sur la distance entre l'arbre planté et le plant d'éricacée le plus proche était toujours très significative $(p<0,001)$.

\section{Concentrations en éléments nutritifs dans le sol et dans les aiguilles}

En octobre 2014, les concentrations en éléments nutritifs dans le sol ne différaient pas significativement entre les traitements de préparation de terrain (tous les $\mathrm{F}_{1,3} \leq 1,02$, tous les $p \geq 0,386$ ) ni entre les essences (tous les $\mathrm{F}_{1,6} \leq 1,74$, tous les $p \geq 0,235)$. De même, nous n'avons décelé aucune différence significative dans les concentrations foliaires en $\mathrm{N}, \mathrm{P}$ et $\mathrm{K}$ des plants mesurés en octobre 2014 dans les parcelles scarifiées ou décapées (tous les $F_{1,3} \leq 8,23$, tous les $p \geq 0,064$ ). Cependant, l'essence a significativement influencé les concentrations foliaires en $\mathrm{P}\left(\mathrm{F}_{1,6}=53,86, p<0,001\right)$ et en $\mathrm{K}\left(\mathrm{F}_{1,6}=14,27\right.$, $p=0,009)$, l'épinette noire ayant des concentrations foliaires en $\mathrm{P}$ et en $\mathrm{K}$ plus élevées que le pin gris ( $\mathrm{P}: 2,2 \pm 0,07 v s .1,4 \pm$ $0,07 \mathrm{~g} / \mathrm{kg} ; \mathrm{K}: 6,3 \pm 0,2$ vs. $5,2 \pm 0,2 \mathrm{~g} / \mathrm{kg})$.

\section{Profondeur de mise en terre et distance à l'andain le plus proche}

Pour l'épinette noire, la profondeur de mise en terre a varié de $-8 \mathrm{~cm}$ à $5 \mathrm{~cm}$, mais na pas eu d'effet significatif sur les taux relatifs de croissance en hauteur et en diamètre des plants dans les parcelles décapées (tous les $p \geq 0,098$; Figs. 2A et 2B). Cependant, elle a eu un effet significatif $(p \leq 0,011)$, mais faible $\left(R^{2} \leq 0,089\right)$ sur cette essence dans les parcelles préparées avec le scarificateur, puisque les taux relatifs de croissance 

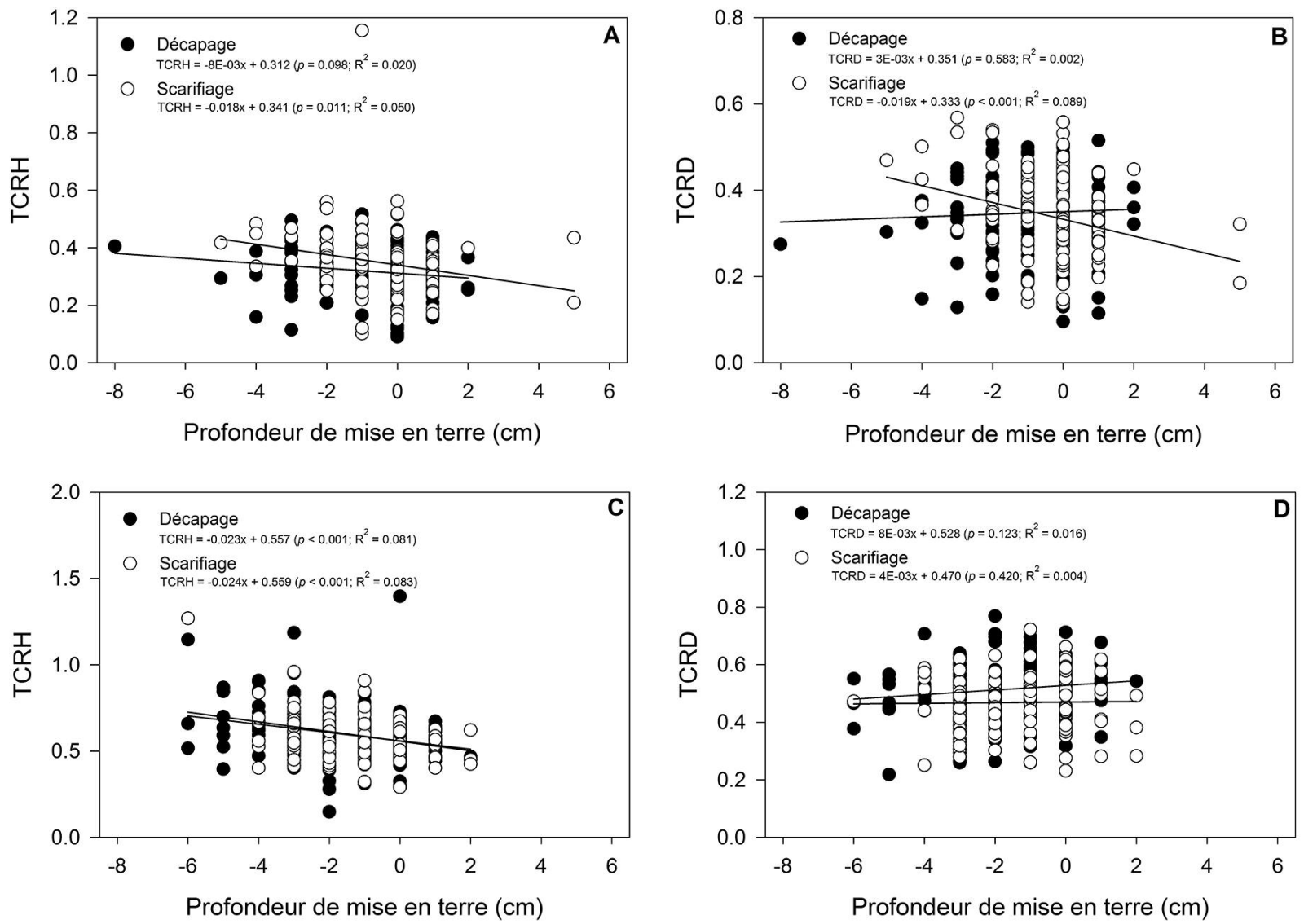

Fig. 2. Relation entre la profondeur de mise en terre et les taux relatifs de croissance en hauteur (TRCH; panneaux A et C) et en diamètre (TRCD; panneaux $B$ et D) sur 3 saisons de végétation pour les plants d'épinette noire (panneaux $A$ et $B$ ) et de pin gris (panneaux $C$ et D). La profondeur correspond à la distance verticale entre le dessus de la carotte de tourbe et la surface du sol au moment de la mise en terre : une valeur négative implique que la carotte a été mise en terre sous le niveau de la surface; une valeur positive implique qu'elle n'a pas été complètement enfouie.

en hauteur (Fig. 2A) et en diamètre (Fig. 2B) ont augmenté avec la profondeur de mise en terre. Pour le pin gris, nous avons observé une relation significative $(p<0,001)$ entre la profondeur de mise en terre et le taux de croissance relative en hauteur, et ce, autant dans les parcelles décapées que dans les parcelles scarifiées (Fig. 2C). La croissance en diamètre du pin gris n’a pas été influencée par la profondeur de mise en terre ( $p \geq 0,123$; Fig. 2D).

Pour les plants mis en terre dans les parcelles décapées, nous n’avons décelé aucun effet significatif $(p \geq 0,142)$ de la distance de mise en terre par rapport à lamas de matière organique le plus proche sur les croissances relatives en hauteur (Fig. 3A) et en diamètre (Fig. 3B) de lépinette noire et du pin gris.

\section{Analyses en composantes principales}

Pour lépinette noire, les 4 premiers axes issus de l'analyse en composantes principales qui avaient des valeurs propres $>1$ expliquaient ensemble $79,7 \%$ de la variance de la matrice. Les variables de concentration en $\mathrm{C}$ dans le sol $(\operatorname{Cos} 2=0,877$; $C T R=27,11)$, de capacité déchange cationique du sol $(\operatorname{Cos} 2=0,817 ; C T R=25,26)$ et de concentration en $\mathrm{N}$ dans le sol $(\operatorname{Cos} 2=0,790 ; C T R=24,42)$ étaient les mieux représentées par le premier axe (valeur propre $=3,235$; Fig. $4 \mathrm{~A}$ ). Pour sa part, le second axe (valeur propre $=2,364$; Fig. 4A) représentait bien le pourcentage de recouvrement en plantes latifoliées $(\operatorname{Cos} 2=0,442 ; C T R=18,68)$, en graminées $(\operatorname{Cos} 2=0,393$; $C T R=16,62)$ et en éricacées $(\operatorname{Cos} 2=0,385 ; C T R=16,28)$, de même que la concentration en $\mathrm{P}$ du sol $(\operatorname{Cos} 2=0,316$; $C T R=16,36$ ). Le troisième axe (valeur propre $=2,02$; pourcentage de la variance expliquée $=15,5$; non présenté) était surtout associé à la concentration foliaire en $\mathrm{N}(\operatorname{Cos} 2=0,664$; $C T R=32,90$; coordonnée $=-0,815)$, au taux relatif de croissance en hauteur $(\operatorname{Cos} 2=0,373$; $C T R=18,47$; coordonnée $=0,611$ ) et au pourcentage de recouvrement en mousses $(\operatorname{Cos} 2=0,346 ; C T R=17,15$; coordonnée $=0,589)$. Finalement, le quatrième axe (valeur propre $=1,689$; pourcentage de la variance expliquée $=13,0$; non présenté) représentait surtout la concentration en $\mathrm{P}$ du sol $(\operatorname{Cos} 2=0,456$; CTR $=27,02$; coordonnée $=0,676)$ et en $\mathrm{K}$ du sol $(\operatorname{Cos} 2=0,370 ; C T R=21,91$; coordonnée $=0,608)$. Lorsqu’elles sont représentées dans le plan formé par les 2 premiers axes (Fig. 4B), les observations associées au scarifiage et au décapage présentaient une séparation incomplète des traitements, avec une superposition des ellipses de confiance à 95 $\%$. Les observations propres aux parcelles décapées étaient davantage associées à des valeurs élevées de concentration de 

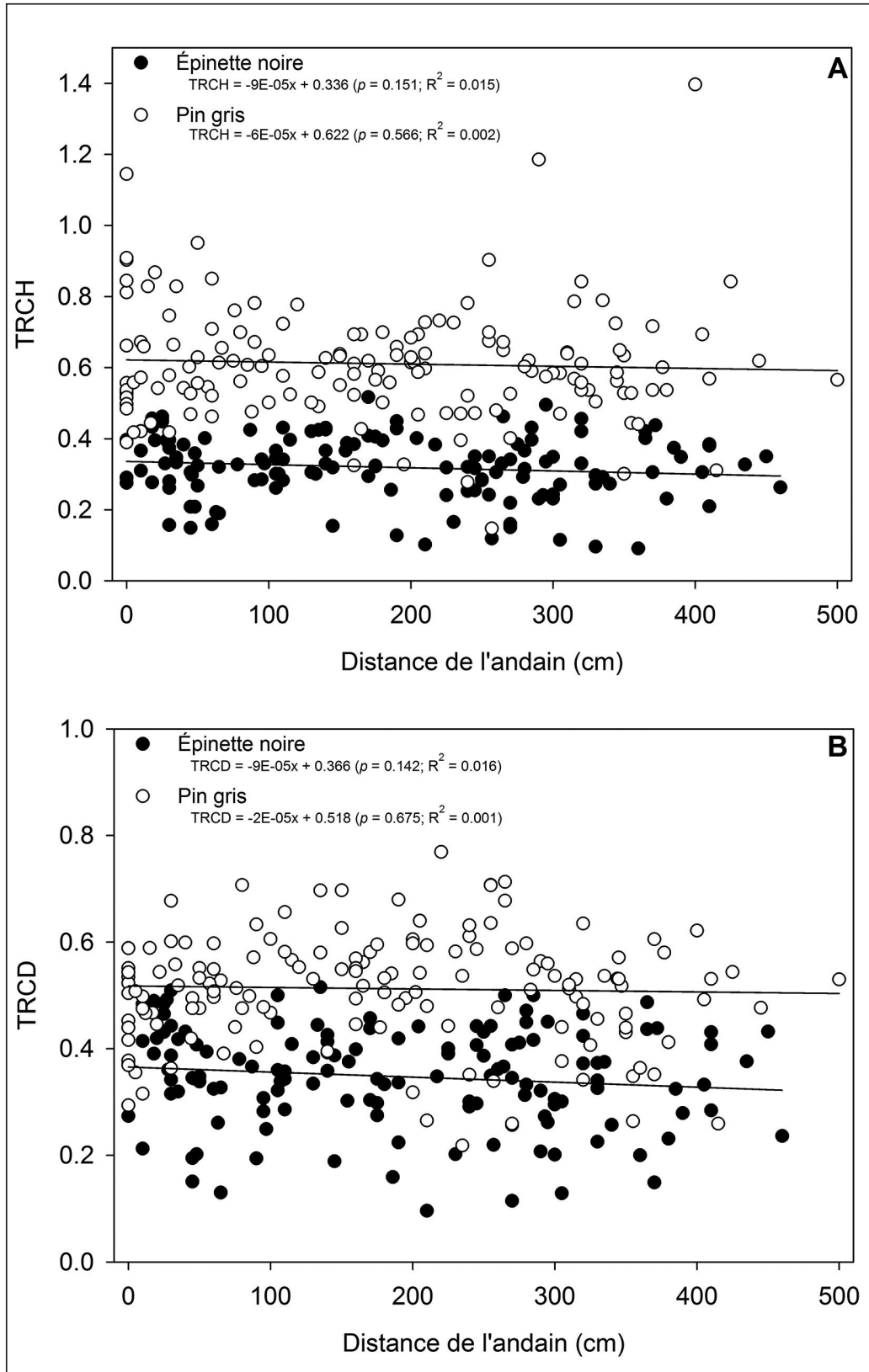

Fig. 3. Relation entre la distance de l'andain le plus proche et les taux relatifs de croissance en hauteur (TRCH; panneau A) et en diamètre (TRCD; panneau B) sur 3 saisons de végétation pour les plants d'épinette noire et de pin gris mis en terre dans les parcelles soumises à un décapage de la matière organique.

$\mathrm{N}$, de concentration de $\mathrm{C}$ et de CEC du sol que les observations propres aux parcelles scarifiées. Celles-ci, pour leur part, étaient davantage associées à des valeurs élevées de concentrations en cations basiques du sol que les observations en provenance des parcelles décapées.

Pour le pin gris, lanalyse en composantes principales des données a permis d'identifier 3 axes avec des valeurs propres $>1$ qui expliquaient ensemble $71,6 \%$ de la variance de la matrice. Le premier axe (valeur propre $=4,089$; Fig. 5A) était fortement associé à la concentration en $\mathrm{C}(\operatorname{Cos} 2=0,653$; $C T R=15,97)$ et en $\mathrm{N}$ du sol $(\operatorname{Cos} 2=0,583$; CTR $=14,25)$, au pourcentage de recouvrement en plantes éricacées $(\operatorname{Cos} 2=0,536 ; C T R=13,10)$ et à la capacité déchange catio- nique $(\operatorname{Cos} 2=0,443 ; C T R=10,83)$. Pour sa part, le second axe (valeur propre $=3,179$; Fig. 5A) capturait essentiellement la variance associée aux concentrations foliaires en $\mathrm{P}(\operatorname{Cos} 2=0,691$; CTR $=21,75)$, $\mathrm{N}(\operatorname{Cos} 2=0,609 ; C T R=19,16)$ et $\mathrm{K}$ $(\operatorname{Cos} 2=0,526 ; C T R=16,53)$. Finalement, le troisième axe (valeur propre $=2,04$; pourcentage de la variance expliquée $=15,7$; non présenté) semblait surtout associé aux pourcentages de recouvrement en plantes latifoliées $(\operatorname{Cos} 2=0,395$; $C T R=19,35$; coordonnée $=0.628)$ et éricacées $(\operatorname{Cos} 2=0,285$; $C T R=13,97$; coordonnée $=0,534)$, de même quà la capacité déchange cationique du sol $(\operatorname{Cos} 2=0,272$; $C T R=13,35$; coordonnée $=-0,522)$. Les distributions des observations associées au décapage et au scarifiage dans lespace défini par les 2 premiers axes (Fig. 5B) montraient un fort chevauchement; les moyennes des coordonnées des observations de chacun des traitements étaient comprises dans l'intervalle de confiance de $95 \%$ de l'autre traitement.

\section{Discussion}

Globalement, les traitements de scarifiage à disques et de décapage de la matière organique ont eu des effets à court terme semblables sur la concentration en éléments nutritifs dans le sol et dans les aiguilles ainsi que sur la croissance de lépinette noire et du pin gris. Ceci, malgré les différences notables que les traitements ont générées quant à la distance et au pourcentage de recouvrement en éricacées, un groupe d’espèces reconnues pour leurs effets négatifs sur la nutrition et la croissance des conifères (de Montigny and Weetman 1990, Titus et al.1995, Thiffault et al. 2004). Malgré leur plus grande abondance dans lenvironnement des plants dans les parcelles scarifiées par rapport aux parcelles décapées, ces espèces semblent avoir peu influencé la disponibilité de ressources clés, comme en témoignent les corrélations presque nulles (Fig. 4) ou positives (Fig. 5) entre le pourcentage de recouvrement en éricacées et les concentrations en $\mathrm{N}$ du sol et la capacité déchange cationique. D'ailleurs, la distribution des observations associées aux 2 traitements dans lespace défini par les 2 premiers axes principaux n'illustre pas de tendances très fortes. Bien que l'abondance des éricacées ait été associée à une plus faible concentration en $\mathrm{P}$ dans le sol, une tendance rapportée dans d'autres travaux (p. ex. Bloom and Mallik 2006), la concentration foliaire en $\mathrm{P}$ pour lépinette noire et le pin gris est demeurée relativement indépendante de la présence des éricacées. Par ailleurs, le scarifiage par sillons crée typiquement des microsites de plantation dont la moitié est constituée de sol minéral exposé (Prévost et Thiffault 2013). Un tel milieu est peu propice à la reproduction végétative du Kalmia (Mallik 1993). 

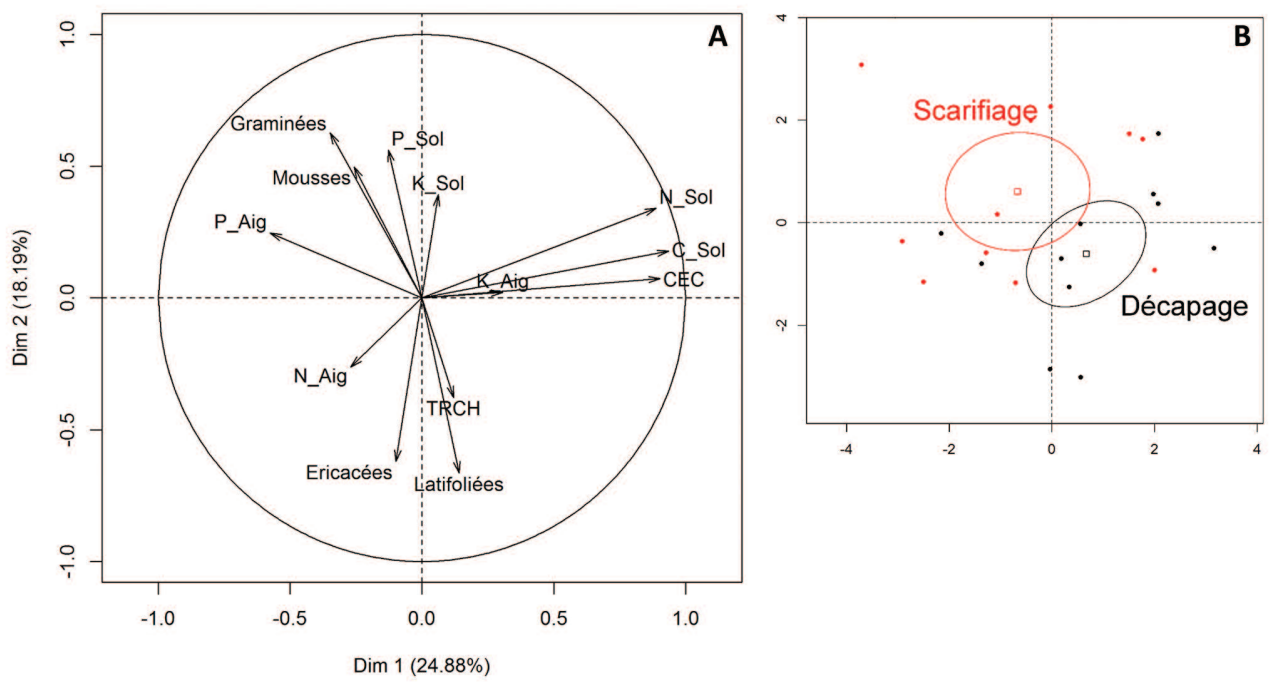

Fig. 4. Analyse en composantes principales illustrant les corrélations entre les caractéristiques du microsite, la proportion de recouvrement des principaux groupes d'espèces, la concentration foliaire en éléments nutritifs et la croissance des plants d'épinette noire (panneau AJ. N_Aig = concentration foliaire en azote; P_Aig = concentration foliaire en phosphore; K_Aig = concentration foliaire en potassium; N_Sol = concentration en azote dans le sol; P_Sol = concentration en phosphore dans le sol; K_Sol = concentration en potassium dans le sol; C_Sol = concentration en carbone dans le sol; $C E C=$ capacité d'échange cationique; TRCH = taux relatif de croissance en hauteur sur 3 saisons de végétation. Le panneau B illustre la distribution des observations associées aux traitements de décapage et de scarifiage dans l'espace défini par les 2 premiers axes; les ellipses représentent les intervalles de confiance à $95 \%$ autour des moyennes des 2 traitements.
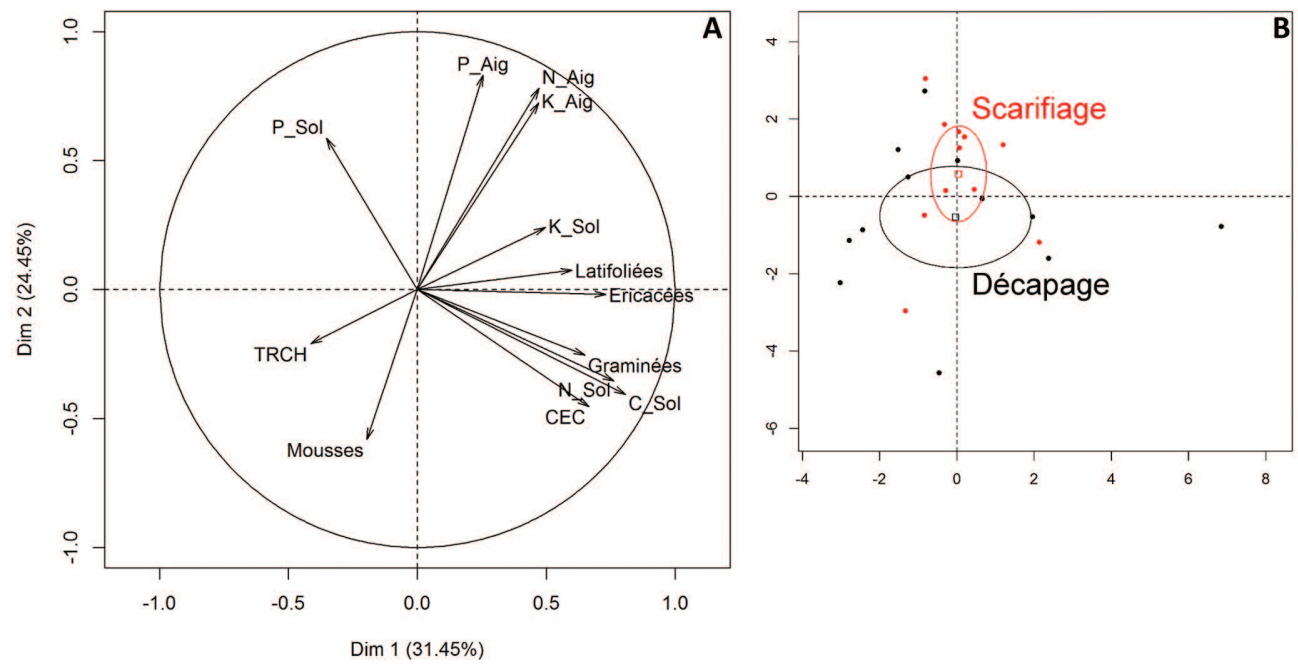

Fig. 5. Analyse en composantes principales illustrant les corrélations entre les caractéristiques du microsite, le recouvrement des principaux groupes d'espèces, la concentration foliaire en éléments nutritifs et la croissance des plants de pin gris (panneau A).

N_Aig = concentration foliaire en azote; P_Aig = concentration foliaire en phosphore; K_Aig = concentration foliaire en potassium; N_Sol = concentration en azote dans le sol; P_Sol = concentration en phosphore dans le sol; K_Sol = concentration en potassium dans le sol; C_Sol = concentration en carbone dans le sol; CEC = capacité d'échange cationique; TRCH = taux relatif de croissance en hauteur sur 3 saisons de végétation. Le panneau B illustre la distribution des observations associées aux traitements de décapage et de scarifiage dans l'espace défini par les 2 premiers axes; les ellipses représentent les intervalles de confiance à $95 \%$ autour des moyennes des 2 traitements. 
Ainsi, les plants mis en terre dans les microsites créés par le scarifiage profitent d'un environnement de croissance partiellement libre de linfluence des espèces éricacées, au moins pour les 3 premières saisons suivant la plantation.

Contrairement à nos prédictions, la croissance des plants mis en terre dans les parcelles décapées n’a pas varié significativement en fonction de la distance des amas de manière organique (essentiellement les andains). Cette absence de relation pourrait être due aux exigences nutritionnelles relativement faibles des conifères (Munson and Timmer 1989) comparativement à celles d'une espèce comme le Prunus pensylvanica L. (Jobidon 1995), pour laquelle une telle relation avait été observée sur des stations soumises à une mise en andains conventionnelle (sans déplacement de la couche organique) (McCavour et al. 2014). Par ailleurs, les caractéristiques de la litière et le climat froid et humide de la forêt boréale ralentissent la minéralisation de la matière organique (Van Cleve et al. 1981, Van Cleve et al. 1990, Scott and Binkley 1997, Pajuste and Frey 2003). Ainsi, il faudra certainement plusieurs années avant que les éléments nutritifs accumulés dans les andains soient remis en circulation (Blumfield et al. 2004). Des suivis à moyen et à long termes de la croissance et du statut nutritif des plants seront nécessaires pour vérifier cette hypothèse. On peut sattendre à ce quau fil du temps, les plants situés au centre des parcelles décapées (donc les plus éloignés des andains) présentent des signes de carences en éléments nutritifs, telle la chlorose des feuilles, que leur croissance ralentisse par rapport à celle des plants situés en bordure des parcelles (près des principaux amas de matière organique) ou même que les systèmes racinaires se développent de manière asymétrique (davantage vers la source déléments nutritifs).

Une mise en terre plus profonde a eu un effet nul ou positif sur la croissance des plants, lequel a varié selon les combinaisons de traitements et dessences. Alors que nous attendions un effet positif marqué de la profondeur de mise en terre dans les parcelles décapées, en raison des variations importantes de température associées à l'enlèvement de l'humus (Heiskanen et al. 2013), c'est dans le traitement de scarifiage que cette variable a eu le plus d'effet, tant pour l'épinette noire que le pin gris. Bien que significative, la relation entre la profondeur de mise en terre et la croissance des plants était tout de même faible, avec un $\mathrm{R}^{2}$ de $9 \%$ dans le meilleur des cas (Fig. 2B). Ce résultat concorde avec ceux de Paquette $e$ t al. (2011) qui ont rapporté que la profondeur de mise en terre n'influençait pas la croissance de lépinette noire, de l'épinette blanche (Picea glauca [Moench.] Voss.) et du pin gris plantés sur des stations boréales, et ce, jusquà 19 ans. Les problèmes de déchaussement sont généralement plus importants sur sols à texture fine, en raison de l'effet du cycle de gel-dégel (Rempel 2010). La texture grossière du sol dans notre site détude pourrait donc expliquer la faible incidence de la profondeur sur la croissance.

De manière prévisible, la croissance du pin gris a largement dépassé celle de lépinette noire. Cet écart reflète vraisemblablement les différences intrinsèques dans les capacités de fixation du carbone d'une essence intolérante à lombre (le pin gris) et d'une autre, plus tolérante à l'ombre (l'épinette noire) (Reich et al. 1998b). Les analyses en composantes principales mettent en perspective ces différences entre essences. Alors que pour l'épinette noire, le taux de croissance relative en hauteur était relativement indépendant de la concentration en $\mathrm{N}$ et de la capacité déchange cationique du sol (à angle droit, respectivement le long des 2 premiers axes; Fig. 4A), ces mêmes variables étaient négativement corrélées pour le pin gris (en directions opposées le long de la première composante; Fig. 5B). Par ailleurs, les concentrations foliaires plus élevées en éléments nutritifs chez lépinette noire illustrent son adaptation à conserver les éléments nutritifs dans les environnements pauvres (Hom and Oechel 1983, Díaz et al. 2004). Par contraste, le pin gris est adapté à réagir rapidement à la fluctuation des ressources disponibles (Díaz et al. 2004). Cependant, la concentration en éléments nutritifs n'informe pas complètement sur le statut nutritif des plants, en raison d'un effet possible de dilution (Timmer 1991). La prise en compte de la masse foliaire aurait permis d'obtenir un portrait plus complet de la réaction des plants à cet égard.

Nos résultats à court terme démontrent que lenlèvement complet de la matière organique par décapage permet létablissement et la croissance initiale de plants de conifères sur des stations boréales à humus épais dominées par les plantes éricacées, sans différence notable par rapport au scarifiage par sillons. À court terme, les effets du décapage sur la nutrition et la croissance des plants peuvent toutefois être masqués par l'effet résiduel des éléments nutritifs exportés depuis la pépinière dans la carotte de tourbe (Idris et al. 2004). L'indépendance entre les concentrations en éléments nutritifs mesurées dans le sol et celles mesurées dans les aiguilles (Fig. 4A et Fig. 5A) illustrent ce découplage entre les quantités de ressources nutritionnelles du microsite et le statut nutritif des plants pendant les premières années suivant la mise en terre. Ces résultats après 3 ans doivent donc être interprétés avec prudence, compte tenu de l'effet potentiel à moyen et long termes de l'exportation de la matière organique associée au décapage. En effet, l'humus forestier constitue un capital important déléments nutritifs, lequel doit être conservé sur les sites afin d'en garantir la productivité à long terme (Prescott et al. 2000). À moyen et à long termes, laccumulation de la matière organique sous forme d'andains linéaires distants de plusieurs mètres risque de priver les arbres situés au centre des parcelles d'un apport suffisant en éléments nutritifs. De plus, l'exposition du sol minéral sur de grandes surfaces favorise lérosion (Merino and Edeso 1999, Merino et al. 2004) et devient une source importante de sédiments dans les cours d'eau (Olarieta et al. 1999), un aspect qui na pas été mesuré dans notre étude. En raison de ce risque, le décapage provoquant l'exposition d'horizons minéraux non fertiles fait l'objet d'un suivi environnemental particulier au Québec (Jetté 2004). D’un autre côté, il est possible que les plantes éricacées envahissent rapidement les sillons dans les parcelles scarifiées, comme le suggère la diminution rapide de la distance entre les arbres plantés et les plantes éricacées dans ces parcelles (Figs. 1A et 1B). Si le taux d'envahissement des microsites scarifiés par le Rhododendron et le Kalmia demeure important, la nutrition et la croissance des plants risquent dêtre affectées (Thiffault et al. 2005). Ainsi, s'il savérait que le décapage de la matière organique offre un avantage significatif par rapport au scarifiage conventionnel à légard de la croissance des plantations sur les stations à humus épais dominées par les éricacées, son utilisation devrait être régie par des modalités qui permettraient d'en limiter les impacts environnementaux. 


\section{Remerciements}

Je remercie Jacques Carignan, Alexandre Fortin-Pelletier, Evelyne Gaillard et plusieurs étudiants dété pour la qualité de leur travail technique, ainsi que Luc Hovington et Michel Fortin pour leur contribution à la planification et la réalisation de cette expérience. Je remercie également Marcel Prévost, Denise Tousignant, Charles Ward, Denis Langlois et deux réviseurs anonymes dont les commentaires judicieux ont permis d'améliorer une version préliminaire de ce manuscrit. Cette étude s'inscrit dans le cadre du projet 142332106 de la Direction de la recherche forestière du ministère des Forêts, de la Faune et des Parcs du Québec et a bénéficié d'un financement du Programme de mise en valeur des ressources du milieu forestier Volet I, catégorie « Expérimentation sylvicole et activités de recherche ou d’acquisition de connaissances ».

\section{Références}

Bates, D., M. Mächler, B.M. Bolker et S.C. Walker. 2015. Fitting linear mixed-effects models using lme4. J. Stat. Soft. 67(1): 1-48. doi:10.18637/jss.v067.i01.

Bloom, R.G. et A.U. Mallik. 2006. Relationships between ericaceous vegetation and soil nutrient status in a post-fire Kalmia angustifolia-black spruce chronosequence. Plant Soil 289(1-2): 211-226. doi:10.1007/s11104-006-9130-3.

Blumfield, T.J., Z.H. Xu et P.G. Saffigna. 2004. Carbon and nitrogen dynamics under windrowed residues during the establishment phase of a second-rotation hoop pine plantation in subtropical Australia. For. Ecol. Manage. 200(1-3): 279-291. doi:10.1016/ j.foreco.2004.07.008.

Bouchard, M., D. Pothier et S. Gauthier. 2008. Fire return intervals and tree species succession in the North Shore region of eastern Quebec. Can. J. For. Res. 38(6): 1621-1633. doi:10.1139/X07-201.

Bradley, R.L., B.D. Titus et J.W. Fyles. 1997. Nitrogen acquisition and competitive ability of Kalmia angustifolia L., paper birch (Betula papyrifera Marsh.) and black spruce (Picea mariana (Mill.) BSP) seedlings grown on different humus forms. Plant Soil 195(2): 209-220. doi:10.1023/A:1004263716346.

Burdett, A. 1990. Physiological processes in plantation establishment and the development of specifications for forest planting stock. Can. J. For. Res. 20(4): 415-427. doi:10.1139/x90-059.

Carter, M.R. (éd). 1993. Soil Sampling and Methods of Analysis. Lewis Publishers and CRC Press LLC, Boca Raton, FL.

de Montigny, L.E. et G.F. Weetman. 1990. The effects of ericaceous plants on forest productivity. Dans The Silvics and Ecology of Boreal Spruce. Édité par Titus, B.D., M.B. Lavigne, P.F. Newton et W.J. Meades. Canadian Forest Service, Forestry Canada, St. John's, Newfoundland. pp. 83-90.

Devine, W.D. et C.A. Harrington. 2007. Influence of harvest residues and vegetation on microsite soil and air temperatures in a young conifer plantation. Agric. For. Meteorol. 145(1-2): 125-138. doi:10.1016/j.agrformet.2007.04.009.

Díaz, S. et al. 2004. The plant traits that drive ecosystems: Evidence from three continents. J. Veg. Sci. 15(3): 295-304. doi:10.1111/ j.1654-1103.2004.tb02266.x.

Dumas. M.T. 2012. Effect of site preparation on Armillaria ostoyae infection and growth of spruce after partial cutting in a Boreal Mixedwood Forest. Forest. Chron. 88(5) : 622-625. doi :10.5558/ tfc2012-115.

Fox, J. et S. Weisberg. 2011. An R Companion to Applied Regression, Second Edition. Sage Publications.

Frey. B.R., V.J. Leiffers, A.D. Munson et P.V. Blenis. 2003. The influence of partial harvesting and forest floor disturbance on nutrient availability and understory vegetation in boreal mixedwoods. Can. J. For. Res. 33(7) : 1180-1188. doi :10.1139/x03-42.

Gauthier, S., M.-A. Vaillancourt, A. Leduc, L.D. Grandpré, D. Kneeshaw, H. Morin, P. Drapeau et Y. Bergeron (éds). 2008. Aménagement écosystémique en forêt boréale. Presses de l'Université du Québec, Québec, QC.
Goulet, F. 2000. Frost heaving of planted tree seedlings in the boreal forest of Northern Sweden. Swedish University of Agricultural Sciences, Department of Silviculture. Reports. 45. Umea, Sweden.

Gradowski, T., D. Sidders, T. Keddy, V.J. Leiffers et S.M. Landhäusser. 2008. Effects of overstory retention and site preparation on growth of planted white spruce seedlings in deciduous and coniferous dominated boreal plains mixedwoods. Forest. Ecol. Manag. 255(11) : 3744-3749. doi :10.1016/j.foreco.2008.03.008.

Gravel, J., M. Prévost et N. Thiffault. 2015. Le scarifiage. Direction de l'aménagement et de l'environnement forestiers et Direction de la recherche forestière, Ministère des Forêts, de la Faune et des Parcs. Publication DAEF2-067-F-18. Québec, QC.

Greene, D.F. et al. 2007. The reduction of organic-layer depth by wildfire in the North American boreal forest and its effect on tree recruitment by seed. Can. J. For. Res. 37(6): 1012-1023. doi:10.1139/x06-245.

Grossnickle, S.C. 2012. Why seedlings survive: influence of plant attributes. New For. 43(5-6): 711-738. doi:10.1007/s11056-0129336-6.

Hébert, F., J.-F. Boucher, D. Walsh, P. Tremblay, D. Côté et D. Lord. 2014. Black spruce growth and survival in boreal open woodlands 10 years following mechanical site preparation and planting. Forestry 87(2): 277-286. doi:10.1093/forestry/cpt052.

Heiskanen, J., T. Saksa et J. Luoranen. 2013. Soil preparation method affects outplanting success of Norway spruce container seedlings on till soils susceptible to frost heave. Silva Fenn. 47(1): article id 893.

Henneb, M., O. Valeria, N.J. Fenton, N. Thiffault et Y. Bergeron. 2015. Mechanical site preparation: Key to microsite creation success on Clay Belt paludified sites. For. Chron. 91(2): 187-196. doi:10.5558/tfc2015-030.

Hom, J.L. et W.C. Oechel. 1983. The photosynthetic capacity, nutrient content and nutrient use efficiency of different needle age classes of black spruce (Picea mariana) found in interior Alaska, USA. Can. J. For. Res. 13(5): 834-839. doi:10.1139/x83-113.

Idris, M., K.F. Salifu et V.R. Timmer. 2004. Root plug effects on early growth and nutrition of container black spruce seedlings. Forest Ecol. Manag. 195(3): 399-408. doi:10.1016/j.foreco.2004.03.005. Inderjit et A.U. Mallik. 1999. Nutrient status of black spruce (Picea mariana [Mill.] BSP) forest soils dominated by Kalmia angustifolia L. Acta Oecol. 20(2): 87-92. doi:10.1016/S1146-609X(99)80020-4.

Jetté, J.-P. 2004. Protéger les sols forestiers par une approche de gestion adaptative. Nat. Can. 128(2): 78-84.

Jobidon, R. 1995. Autécologie de quelques espèces de compétition d'importance pour la régénération forestière au Québec. Revue de littérature. Direction de la rechereche forestière, Ministère des Ressources naturelles. Mémoire de recherche forestière 117. Québec. QC. Lazaruk, L.W., S.E. Macdonald et G. Kernaghan. 2008. The effect of mechanical site preparation on ectomycorrhizae of planted white spruce seedlings in conifer-dominated boreal mixedwood forest. Can. J. For. Res. 38(7) : 2072-2079. doi :10.1139/x08-035.

Lê, S., J. Josse et F. Husson. 2008. FactoMineR: An R package for multivariate analysis. J. Stat. Soft. 25(1): 1-18. doi:10.18637/ jss.v025.i01.

LeBel, P., N. Thiffault et R.L. Bradley. 2008. Kalmia removal increases nutrient supply and growth of black spruce seedlings: An effect fertilizer cannot emulate. For. Ecol. Manage. 256(10): 1780-1784. doi:10.1016/j.foreco.2008.02.050.

Lieffers, V.J., C. Messier, P.J. Burton, J.-C. Ruel et B.E. Grover. 2003. Nature-based silviculture for sustaining a variety of boreal forest values. In : Towards Sustainable Management of the Boreal Forest. Édité par Burton, P.J., C. Messier, D.W. Smith et W.L. Adamowicz. NRC Research Press, Ottawa, ON. pp. 481-530.

Mallik, A.U. 1993. Ecology of a forest weed of Newfoundland: vegetative regeneration strategy of Kalmia angustifolia. Can. J. Bot. 71(1): 161-166. doi:10.1139/b93-018.

Margolis, H.A. et D.G. Brand. 1990. An ecophysiological basis for understanding plantation establishement. Can. J. For. Res. 20(4): 375-390. doi:10.1139/x90-056. 
McCavour, M.J., D. Paré, C. Messier, N. Thiffault et E. Thiffault. 2014. The role of aggregated forest harvest residue in soil fertility, plant growth, and pollination services. Soil Sci. Soc. Am. J. 78(S1): S196-S207. doi:10.2136/sssaj2013.08.0373nafsc.

Merino, A. et J.M. Edeso. 1999. Soil fertility rehabilitation in young Pinus radiata D. Don. plantations from northern Spain after intensive site preparation. For. Ecol. Manage. 116(1-3): 83-91. doi:10.1016/S0378-1127(98)00444-7.

Merino, A., A. Fernández-López, F. Solla-Gullón et J.M. Edeso. 2004. Soil changes and tree growth in intensively managed Pinus radiata in northern Spain. For. Ecol. Manage. 196(2-3): 393-404. doi:10.1016/j.foreco.2004.04.002.

Munson, A.D. et V.R. Timmer. 1989. Site specific growth and nutrition of planted Picea mariana in the Ontario Clay Belt. II. Effects of nitrogen fertilization. Can. J. For. Res. 19(2): 171-178. doi:10.1139/ x89-024.

Munson, A.D., H.A. Margolis et D.G. Brand. 1993. Intensive silvicultural treatment: Impacts on soil fertility and planted conifer response. Soil Sci. Soc. Am. J. 57(1): 246-255. doi:10.2136/ sssaj1993.03615995005700010043x.

Newmaster, S.G., W.C. Parker, F.W. Bell et J.M. Paterson. 2007. Effect of forest floor disturbances by mechanical site preparation on floristic diversity in a central Ontario clearcut. Forest. Ecol. Manag. 246(2-3) : 196-207. doi :10.1016/j.foreco.2007.03.058.

Nyland, R.D. 2002. Silviculture: Concepts and Applications. Second Edition. Waveland Press, Inc., Long Grove, Illinois.

Olarieta, J.R., G. Besga, R. Rodríguez, A. Usón, M. Pinto et S. Virgel. 1999. Sediment enrichment ratios after mechanical site preparation for Pinus radiata plantation in the Basque Country. Geoderma 93(3-4): 255-267. doi:10.1016/S0016-7061(99)00063-4.

Pajuste, K. et J. Frey. 2003. Nitrogen mineralisation in podzol soils under boreal Scots pine and Norway spruce stands. Plant Soil 257(1): 237-247. doi:10.1023/A:1026222831694.

Paquette, A., J. Girard et D. Walsh. 2011. Deep planting has no short- or long-term effect on the survival and growth of white spruce, black spruce, and jack pine. North. J. Appl. For. 28(3): 146-151. Prescott, C.E., D.G. Maynard et R. Laiho. 2000. Humus in northern forests: friend or foe? For. Ecol. Manage. 133(1-2): 23-36. doi:10.1016/S0378-1127(99)00295-9.

Prévost, M. 1992. Effets du scarifiage sur les propriétés du sol, la croissance des semis et la compétition : revue des connaissances actuelles et perspectives de recherches au Québec. Ann. Sci. For. 49(3): 277-296. doi:10.1051/forest:19920306.

Prévost, M. et D. Dumais. 2003. Croissance et statut nutritif de marcottes, de semis naturels et de plants dépinette noire à la suite du scarifiage : résultats de 10 ans. Can. J. For. Res. 33(1): 2097-2107. doi:10.1139/X03-130.

Prévost, M. et N. Thiffault. 2013. La préparation de terrain. Dans Le guide sylvicole du Québec. Tome 2. Les concepts et l'application de la sylviculture. Édité par Larouche, C., F. Guillemette, P. Raymond et J.-P. Saucier. Les Publications du Québec, Québec, QC. pp. 134-157. R Core Team. 2013. R: A language and environment for statistical computing. R Foundation for Statistical Computing, Vienna, Austria. Reich, P.B., M.B. Walters, M.G. Tjoelker, D. Vanderklein et C. Buschena. 1998a. Photosynthesis and respiration rates depend on leaf and root morphology and nitrogen concentration in nine boreal tree species differing in relative growth rate. Funct. Ecol. 12(3): 395-405. doi:10.1046/j.1365-2435.1998.00209.x.

Reich, P.B., M.G. Tjoelker, B. Walters, D. Vanderklein et C. Buschena. 1998b. Close association of RGR, leaf and root morphology, seed mass and shade tolerance in seedlings of nine boreal tree species grown in high and low light. Funct. Ecol. 12(3): 327-338. doi:10.1046/j.1365-2435.1998.00208.x.

Rempel, A.W. 2010. Frost heave. J. Glaciol. 56(200): 1122-1128.

Sahlén, K. et F. Goulet. 2002. Reduction of frost heaving of Norway spruce and scots pine seedlings by planting in mounds or in humus. New For. 24(3): 175-182. doi:10.1023/A:1021378228524.

Salonius, P.O. 1983. Effects of organic-mineral mixtures and increa- sing temperature on the respiration of coniferous raw humus material. Can. J. For. Res. 13(1): 102-107. doi:10.1139/x83-015.

Saucier, J.P., A. Robitaille et P. Grondin. 2009. Cadre bioclimatique du Québec. Dans Manuel de foresterie, 2nd edition. Édité par Doucet, R. et M. Côté. Ordre des ingénieurs forestiers du Québec, Éditions Multimondes, Québec, QC. pp. 186-205.

Scott, N.A. et D. Binkley. 1997. Foliage litter quality and annual net $\mathrm{N}$ mineralization: Comparison across North American forest sites. Oecologia 111(2): 151-159. doi:10.1007/s004420050219.

Soil Classification Working Group. 1998. The Canadian System of Soil Classification. 3rd ed. Agriculture and Agri-Food Canada. Publication 1646. Ottawa, ON.

Sutton, R.F. 1995. Advantages of deep planting black spruce. Natural Resources Canada, Canadian Forest Service. Technical Note 50. Sault Ste. Marie, ON.

Thiffault, N. et P. Grondin. 2003. Envahissement des parterres de coupe par les éricacées. Dans : Les enjeux de biodiversité relatifs à la composition forestière, P. Grondin et A. Cimon, coordonnateurs. Ministère des Ressources naturelles, de la Faune et des Parcs, Direction de la recherche forestière et Direction de l'environnement forestier. Québec, QC.

Thiffault, N. et R. Jobidon. 2006. How to shift unproductive Kalmia angustifolia - Rhododendron groenlandicum heath to productive conifer plantation. Can. J. For. Res. 36(10): 2364-2376. doi:10.1139/ x06-090.

Thiffault, N., B.D. Titus et A.D. Munson. 2004. Black spruce seedlings in a Kalmia-Vaccinium association: microsite manipulation to explore interactions in the field. Can. J. For. Res. 34(8): 1657-1668. doi:10.1139/x04-046.

Thiffault, N., B.D. Titus et A.D. Munson. 2005. Silvicultural options to promote seedling establishment on Kalmia-Vacciniumdominated sites. Scand. J. For. Res. 20(2): 110-121. doi:10.1080/ 02827580510008356.

Thiffault, N., G. Picher et I. Auger. 2012. Initial distance to Kalmia angustifolia as a predictor of planted conifer growth. New For. 43(56): 849-868. doi:10.1007/s11056-012-9324-x.

Thiffault, N., V. Roy, J. Ménétrier, G. Prégent et A. Rainville. 2013. La plantation. Dans Le guide sylvicole du Québec. Tome 2. Les concepts et l'application de la sylviculture. Édité par Larouche, C., F. Guillemette, P. Raymond et J.-P. Saucier, Québec, QC. pp. 196-225. Thiffault, N., P. Grondin, J. Noël et V. Poirier. 2015. Ecological gradients driving the distribution of four Ericaceae in boreal Quebec, Canada. Ecol. Evol. 5(9): 1837-1853. doi:10.1002/ece3.1476.

Timmer, V.R. 1991. Interpretation of seedling analysis and visual symptoms. In: Mineral nutrition of conifer seedlings. Édité par Van Den Driessche, R. CRC Press, Boston, MA. pp. 113-134.

Titus, B.D., S.S. Sidhu et A.U. Mallik. 1995. A summary of some studies on Kalmia angustifolia L.: A problem species in Newfoundland forestry. Natural Resources Canada, Canadian Forest Service. Information Report N-X-296. St-John's, NFL.

Trottier-Picard, A., E. Thiffault, A. DesRochers, D. Paré, N. Thiffault et C. Messier. 2014. Amounts of logging residues affect planting microsites: A manipulative study across northern forest ecosystems. Forest Ecol. Manag. 312: 203-215. doi:10.1016/j.foreco.2013.10.004. Van Cleve, K., R. Barney et R. Schlentner. 1981. Evidence of temperature control of production and nutrient cycling in two interior Alaska black spruce ecosystems. Can. J. For. Res. 11(2): 259-274. doi:10.1139/x81-035.

Van Cleve, K., W.C. Oechel et J.L. Hom. 1990. Response of black spruce (Picea mariana) ecosystems to soil temperature modification in interior Alaska. Can. J. For. Res. 20(9): 1530-1535. doi:10.1139/x90-203. Walinga, I., J.J. van der Lee, V.J.G. Houba, W. van Vark et I. Novozamsky (eds.). 1995. Plant Analysis Manual, Dordrecht, The Netherlands.

Ward, C., D. Pothier et D. Paré. 2014. Do boreal forests need fire disturbance to maintain productivity? Ecosystems 17(6): 1053-1067. doi:10.1007/s10021-014-9782-4.

Yee, T.W. 2008. The VGAM Package. R News 8(2): 28-39. 